\title{
REAL TIME CONTROL OF THE OBSERVING PROGRAM OF AN ORBITING SOLAR OBSERVATORY
}

\author{
E. M. REEVES, M.C. E. HUBER, G. L. WITHBROE and R. W. NOYES \\ Harvard College Observatory, Cambridge, Mass., U.S.A.
}

\section{Introduction}

This paper departs somewhat from the usual interpretation of the subject of this symposium, in which instrumental techniques normally deal with diverse aspects of experiment design or construction. Rather, we will concern ourselves here with some innovations in the ground control optimization of the modes of data acquisition once an instrument is in orbit. Although the fullest possible utilization of the data gathering capability of a device is of vital interest to the success of the experiment and the mission, the subject has not been discussed at length in the open forum of scientific meetings. The planning of the data gathering and processing aspects of a mission must receive as careful attention as instrument design and test if the characteristics of the instrument are to be fully exploited.

In this paper we discuss a specific example, namely, the Harvard experiment on OSO 6, although the elements of our problem apply to many types of experiments in space, particularly those in which the experimenter is required to choose from among many possible experiment modes in direct response to the telemetered data and the specific conditions at the source (in this case the Sun). Extensive preflight planning of diverse series of data gathering patterns is assumed to have been fulfilled, and the purpose of the real-time or near-real time mission control is to complete as many of the objectives as possible, in the fashion best suited to conditions on the Sun at the time, and to assess the quality of the data gathered in order to determine whether the observations are of the necessary quality and quantity to satisfy the observing objective.

\section{Instrument Description}

The Harvard College Observatory ( $\mathrm{HCO}$ ) experiment was mounted in the sail portion of the OSO 6 satellite and launched in August 1969. Light from the Sun entered through a square aperture on the front of the instrument and was imaged on the entrance slit of the spectrometer by an iridium-coated off-axis parabolic mirror. The entrance slit selected a 35-arc sec region of the Sun and determined both the spatial and spectral resolution of the instrument. An original grating ruled in gold with 1800 lines/mm was mounted in a Johnson-Onaka configuration. An open-structured crossed electric and magnetic-field multiplier with tungsten photocathode was located behind the single fixed exit slit. and was operated in a pulse counting mode. Pulses from the multiplier were counted in a binary counter and the data were both trans- 
mitted to the ground and stored by on-board tape recorders with a capacity equivalent to one orbit.

The grating was pivoted in discrete steps of $0.1 \AA$ with a stepping motor and cam arrangement; an entire spectral scan from 280 to $1390 \AA$ consisted of about 10000 steps and required 16 min to complete. The size of the exit slit of the spectrometer was deliberately chosen to exceed the width of the entrance slit by 12 steps of the grating. A grating reference system allowed the grating to be stopped at any step in the entire wavelength region, by stored command from the ground, thus leaving the instrument in a monochromator mode. The main instrument features and operation are similar to those of our experiment on OSO 4; a more detailed description can be found elsewhere (Reeves and Parkinson, 1970; Macar et al., 1970).

The OSO 6 satellite provided the capability to perform spatial scans of the solar disc, thus enabling the instrument to obtain spectroheliograms in the wavelength setting of the grating. The spatial scan characteristics are given in Figure 1, which shows the sizes of the two raster patterns in comparison with the size of the solar disc. The large raster had the following characteristics:

$\begin{array}{ll}\text { raster size } & 46 \times 46 \overbrace{\mathrm{min}^{2}} \\ \text { no. of lines } & 64 \\ \text { time per line } & 7.68 \mathrm{~s} \\ \text { raster time } & 8 \mathrm{~m} 11.5 \mathrm{~s} \\ \text { rasters/orbit } & 6\end{array}$

This large raster was used to survey conditions on the entire Sun in different series of wavelengths, and was also used to integrate the intensity from the whole Sun for certain specialized tasks such as photometric calibration.

Since the time resolution of the large raster was insufficient to follow transient events such as flares, an optional smaller raster was provided, which had the following characteristics:

$\begin{array}{ll}\text { raster size } & 7 \times 7.5 \overbrace{\mathrm{min}^{2}} \\ \text { no. of lines } & 16 \\ \text { time per line } & 1.92 \mathrm{~s} \\ \text { raster time } & 30.7 \mathrm{~s} \\ \text { rasters/orbit } & 98\end{array}$

The small raster pattern could be centered anywhere within the boundaries of the large raster pattern to obtain the time-dependent spatial variations within active regions on the disc or at the limb, or to observe the structure and development of surges, prominences, etc. Only a single wavelength setting of the instrument could be utilized between access times of commanding ground stations, so that investigating a given feature as a function of wavelength (say a series of lines of increasing temperature of formation) could take many successive orbits to complete.

The combination of instrument and spacecraft characteristics also provided the ability to obtain the spectra of selected features on the solar disc. The spacecraft could position the optical axis of the instrument anywhere within the spatial extent of the 


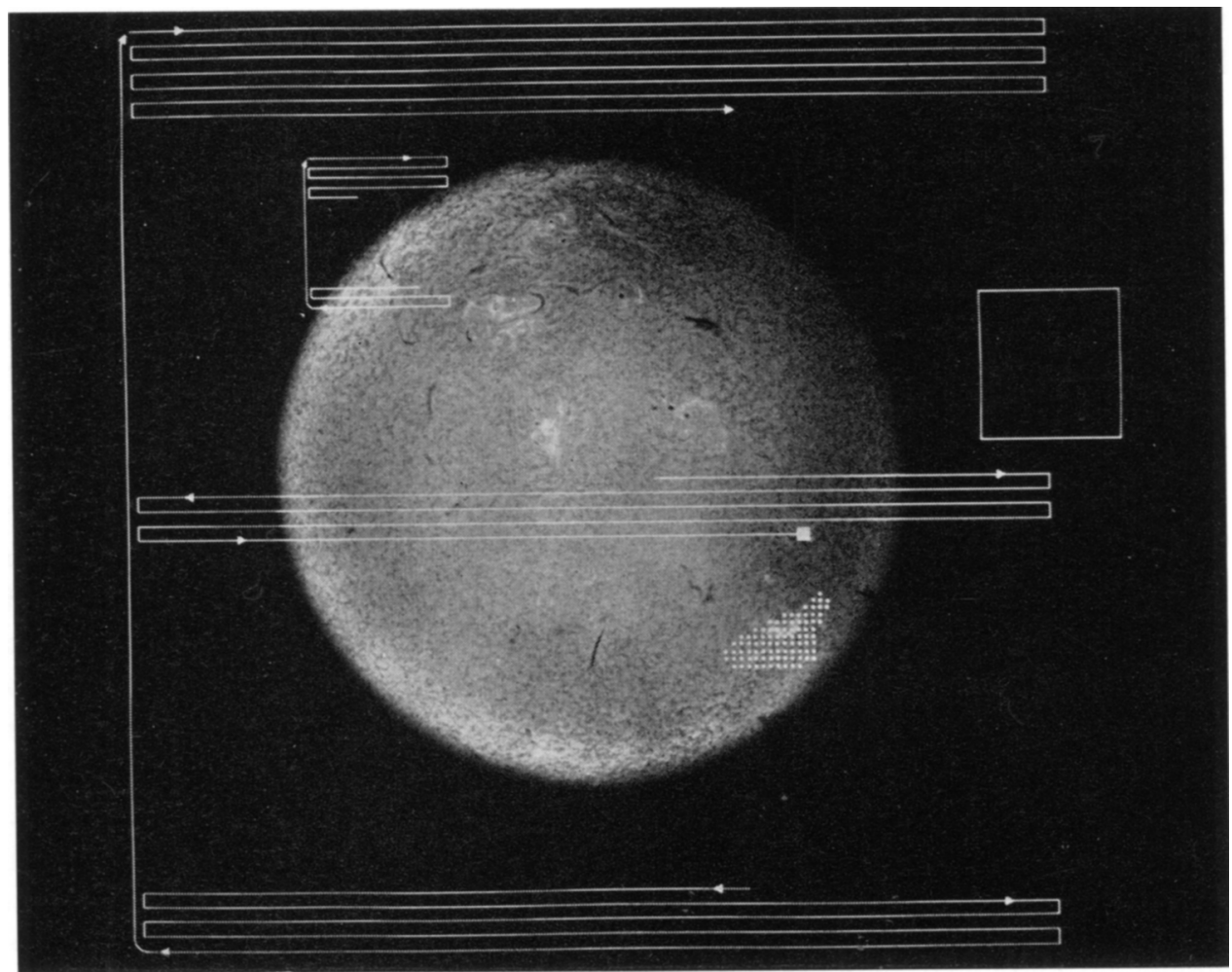

Fig. 1. OSO 6 raster patterns and offset pointing grid.

large raster at discrete positions approximately 20 arc sec apart, set by command of the coordinates of the position matrix from the ground. This offset point matrix of positions is shown schematically in Figure 1 by a series of dots on the bottom right portion of the solar disc. Again the object was to obtain the spectra from selected features on the Sun arising from a number of phenomena such as active regions, filaments, holes and prominences and, for studies such as the center-to-limb variations, of spectral lines and continua and the investigation of the vertical structure of active regions when these could be observed 'edge on' at the solar limb.

\section{Communication Link}

The instrument and spacecraft combination had the capacity for a choice of raster sizes and frequency, and the capability to obtain spectra of selected features on the disc; it was therefore necessary to have a rather extensive communication system to transmit data from the spacecraft to Harvard and to communicate the necessary commands from the scientists back to the spacecraft.

The OSO 6 orbit had a 30-degree inclination at a height of $500 \mathrm{~km}$. In such an 
orbit the period for solar observation is the daylight portion of the orbit, which is about $60 \mathrm{~min}$ long, followed by a period of $30 \mathrm{~min}$ when the spacecraft is in darkness. Fortunately the spacecraft command system was such that commands could be sent either during the daylight portion of the orbit for immediate implementation, or at night for automatic execution at the following sunrise.

The number of available ground stations allowed the tape recorders to be dumped and commands to be transmitted at least once per revolution. The two ground stations at Ft. Myers and at Santiago were each capable of accepting about 5-6 successive passes of the satellite, so that between them they could cover 10-12 of the 16 orbits per day. The remaining orbits could be covered by intermediate South American ground stations and by Johannesburg and Orroral. The latter two stations also served the very important function of giving access to the spacecraft more than once per revolution, which significantly improved the efficiency of the data taking process.

One of the most important aspects of the satellite control process was the receipt and display of the data at Harvard Observatory. When the tape recorders were dumped to the ground station the entire $90 \mathrm{~min}$ of data were recorded on the ground in $5 \mathrm{~min}$. From Ft. Myers, the orbit's data were then played back into a dedicated telephone line directly into a computer at Goddard Space Flight Center, which stripped out the data from the Harvard experiment, changed the format, and again transmitted the coded data to Harvard College Observatory over a second dedicated telephone line. The data terminal at HCO consisted of a special high-speed printer driven by the computer at GSFC, and a tape recorder to record the incoming data for replay if required or in the event of a printer malfunction. A teletype unit received data from ESSA in Colorado to provide an up-to-date status report on solar conditions. Also concluded in the communication system was a facsimile copying machine that could transmit hard copies of written instructions and information over a telephone line between GSFC and HCO.

The data transmitted by the satellite in real time, that is (the periods before and after tape recorder playback when the spacecraft was within range of the ground station) were received and displayed at $\mathrm{HCO}$ instantaneously. This real-time period amounted to about 5 min of data in which nominal operation of the instrument could be checked from the engineering and scientific data; if necessary, commands shutting down the experiment could have been initiated and sent during the $10 \mathrm{~min}$ of the pass. The first tape-recorded data from the orbit were customarily received at $\mathrm{HCO}$ within $20 \mathrm{~min}$ of the ground station pass, and the entire orbit's data were received in $45 \mathrm{~min}$ from stations such as Ft. Myers, Rosman (N.C.) and Orroral (Australia). From the other ground stations only one quarter of the daylight data from the orbit would be available to $\mathrm{HCO}$ because of reduced transmission capabilities from remote sites.

\section{HCO Operations}

Clearly the control of an orbiting observatory facility, such as OSO 6, requires the capability to respond to the data from the instrument, the operating condition of the 
instrument, and the state of activity of the Sun. The operation at the Harvard end of the data link was devised to attempt to obtain the best data possible during what is usually a rather limited instrument lifetime of under one year. Although this may seem a long time, it is not any too great to fully exploit the capabilities of an EUV spectrometer and spectroheliometer, especially when the vagaries of solar conditions are considered.

The focal point of the instrument control exercise was a daily meeting, attended by as many of the solar scientific staff as possible, to review the previous day's data and assess the degree to which that particular day's observing objectives had been achieved. In addition assessing the immediate objectives, they also reviewed the degree to which the preflight observing objectives had been achieved. The very great importance of rather extensive preplanned programs of observations has already been mentioned. For maximum effectiveness the programs must be established and ranked for priority before the instrument is launched. Although priorities are altered during the course of the mission, the process of establishing specific priorities of observations is very valuable in the discussions that take place in the heat of a real operation. Establishing objectives and priorities significantly aids the cataloguing of observations and reduces unnecessary repetition. With an unmanned satellite, extensive preflight planning never prevents a response to solar conditions but rather allows a simpler approach to generating the detailed step-by-step command sequences to achieve the observations, since once an objective has been decided upon, the implied sequence of observations is already substantially available. Whimsey is not excluded from the choice of observations, nor is the generation of completely new and unanticipated observational ideas based only on new data received. These possibilities are decidedly easier to include, because much of the background implications of a change in sequence have already been thought through.

Available to the members of the daily planning session, then, were the state of completion of the preplanned objectives, the previous few days' specific observations, the conditions on the Sun, and the solar forecasts available from ESSA through a teletype link in the data room, $\mathrm{H} \alpha$ and $\mathrm{CaK}$ photographs of the few previous days (kindly provided by SAC Peak and McMath Hulburt Observatories) and, weather permitting, a current $\mathrm{H} \alpha$ photograph. A small $\mathrm{H} \alpha$ telescope equipped with a solid etalon FabryPérot filter was set up on the Observatory roof. Although seeing conditions in Cambridge are not of the highest quality, it was most useful to be able to observe the Sun directly and to have a truly current $\mathrm{H} \alpha$ photograph to compare with the EUV spectroheliograms. The development of these filters for our experiment on the manned Apollo Telescope Mount program has greatly simplified the ease of construction of small $\mathrm{H} \alpha$ telescopes. For a more detailed discussion of the narrow band filters the reader is referred elsewhere (Title, 1970; Carson et al., 1968; Reeves et al., 1968).

Once the observing objectives for the next period of $24-48 \mathrm{~h}$ had been established at the daily discussion, the program was executed on a shift basis by teams consisting of a duty scientist. engineer, and technician. Their tasks were to take the required objectives and coordinate the command times with the available ground stations. 
considering such problems as the relative periods required to complete each observation (such as large raster, small raster, or wavelength scan), the available ground stations, and the day-night periods in relation to the ground stations. When the required sequences of steps were established, a computer was used to look up the precise sequence of coded commands and the required format. These commands were addressed with the appropriate ground station and transmittal times, and a 'telecopier' was used to send a copy of the computer output command request to GSFC via telephone. This procedure considerably reduced errors in copying the commands by hand from tables, and in formulating and transmitting telegrams. It insured that the OSO Control Center at GSFC had a correct copy of our requested commands. GSFC control center would then check these commands, coordinate them with their spacecraft instrument command requirements and make the necessary arrangements with the ground stations to secure the down-link data dumps and up-link commands.

The duty scientist and engineer would also check the data as it came in each orbit, plan recovery procedures when some error in operation was encountered, or initiate a new observing program if a sudden change in solar conditions was reported or discerned in the Harvard data.

\section{Examples of Operational Sequences}

The next few figures will illustrate a typical process of data acquisition to obtain wavelength scans of selected features on the solar disc. Figure 2 shows the printer output of a large raster as a matrix of numbers for the $1334 \AA$ line of C II. Each number is a coded indication of the instrument count rate at that spatial element in the matrix. Contour lines were drawn on the computer printout and were then coloured to make the structures more easily seen. The approximate location of the active region was known from both the ground-based $\mathrm{H} \alpha$ pictures and from previous large rasters in other wavelengths. The approximate location of the srnall raster was already decided when the first large raster was received at HCO. The small rasters of the region were sufficiently well centered to contain the active region, and a printer output of the small raster is shown in Figure 3, as it was used in the operation. From the small raster pictures the exact offset pointing positions for, say, a series of wavelength scans at a number of locations within the active regions could be specified within the 20 arc sec grid positions available for offset pointing. Figure 4 shows a portion of a wavelength scan from a quiet location near the active region as, for example, the first in a sequence of wavelength scan positions stepping across the active region to explore its structure. The wavelength scan was presented as a sequence of five digit numbers, one for every other step of the grating. To make a simple display for the printer, twelve of these numbers (each the average of two successive data words) were printed on each line of the high speed printer; the remainder of the line contained two characters in positions corresponding to the average intensity of the first and last six numbers in that line. A rough spectral scan could then be constructed for quick analysis by a 'follow-the-dots' procedure. with the actual numbers present for any detailed analysis desired. Some of 


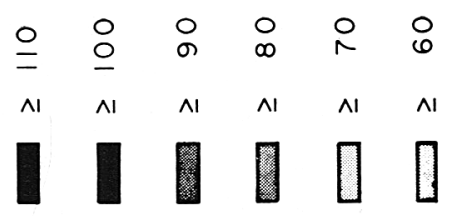

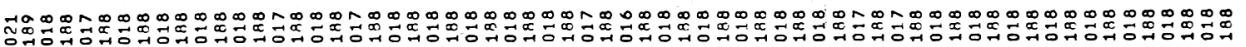

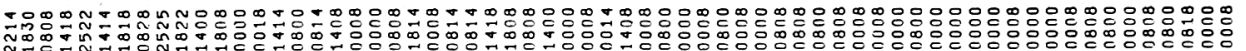

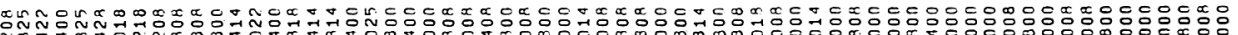

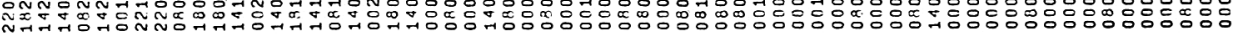

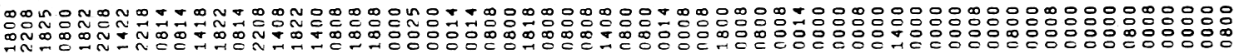
议

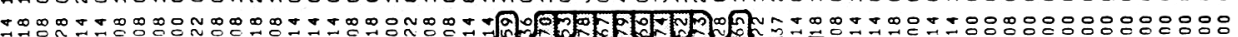

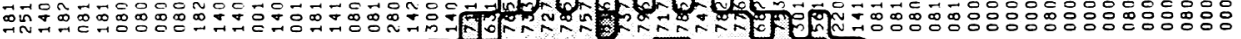

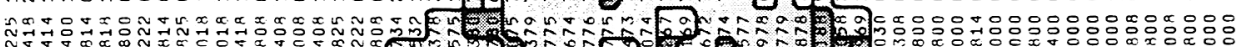

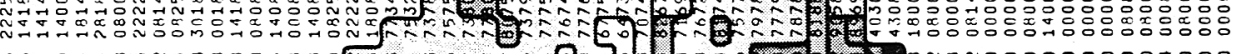
mơ: 等

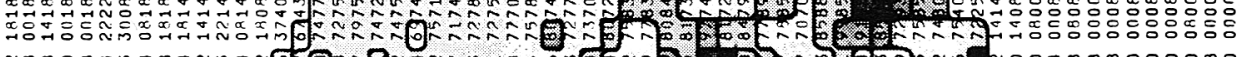
Nom: món

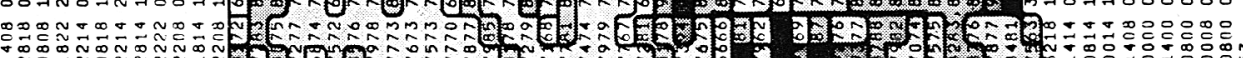

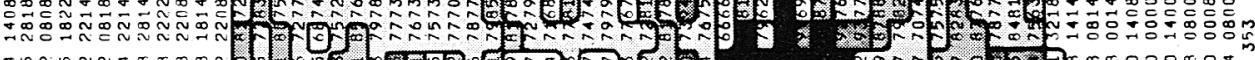

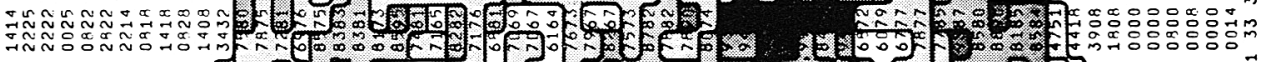

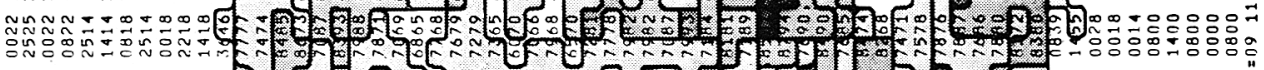

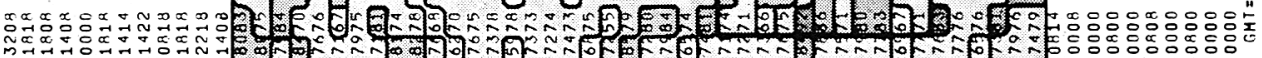

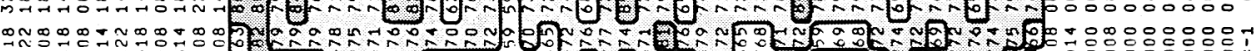

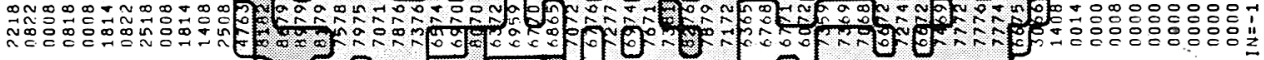

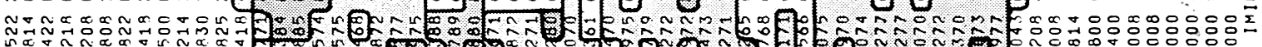

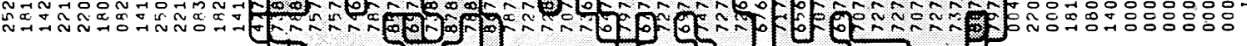

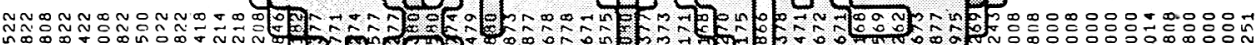
Nom

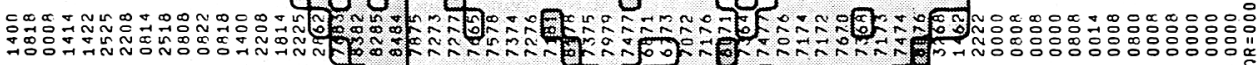

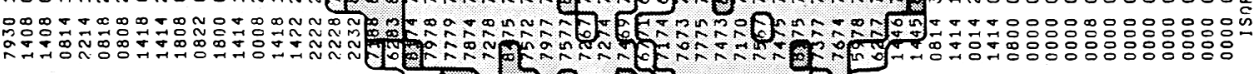

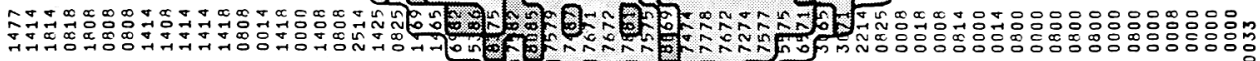

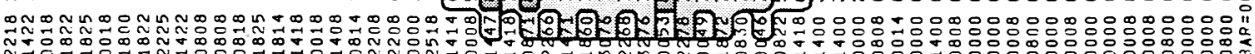

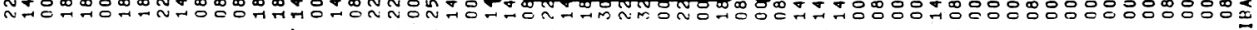

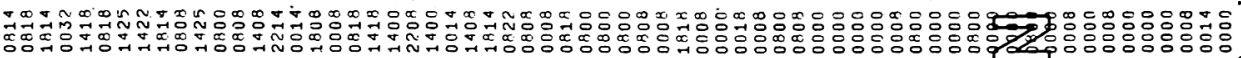

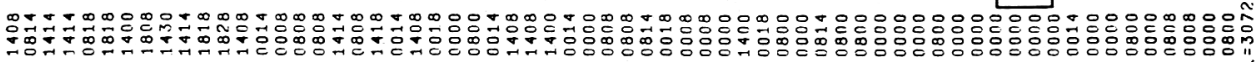
순

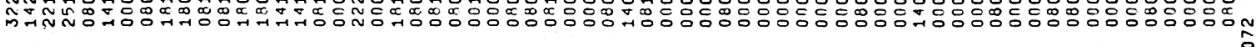

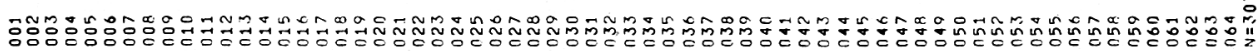




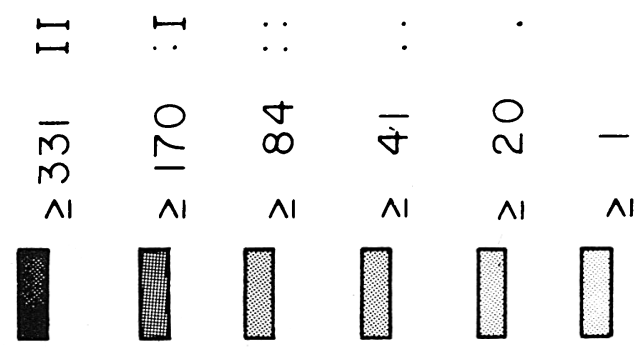

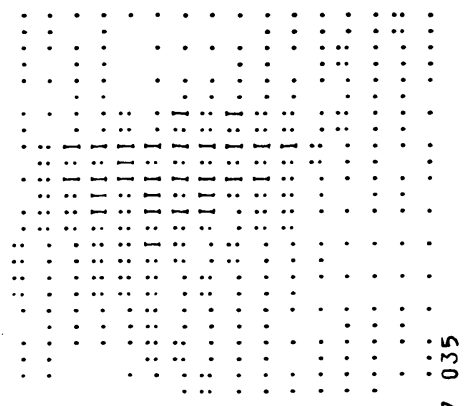

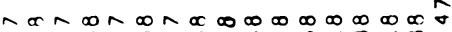

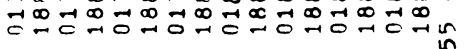

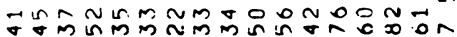

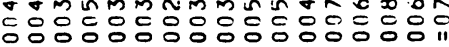

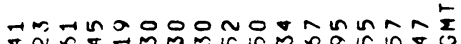

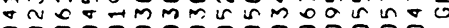
응응응을읃읃읃영응응응응읃응을

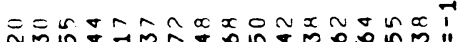

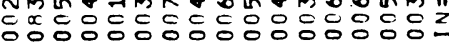

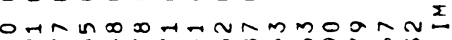

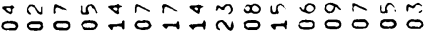

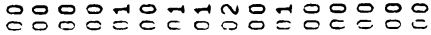
ncn-H-anmouso-co光 mosodinn

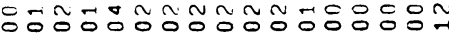

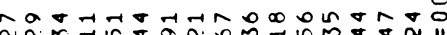

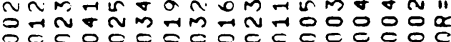

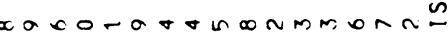

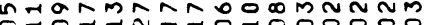

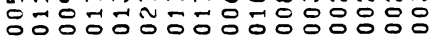

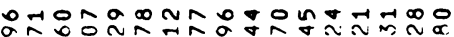

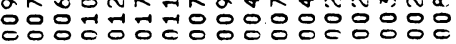

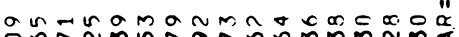

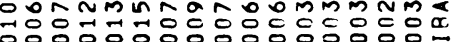
m

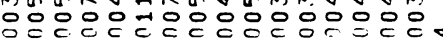
mna

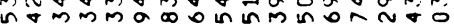
등등등ㄷㅇㅇㅇㅇㅇㅇㅇㅇㅇㅇㅇㅇㅇㅇㅇㅇㅇ은 mon

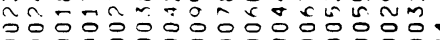
응읃응읃응응읃응응응읃응응응

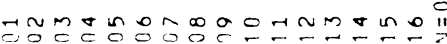

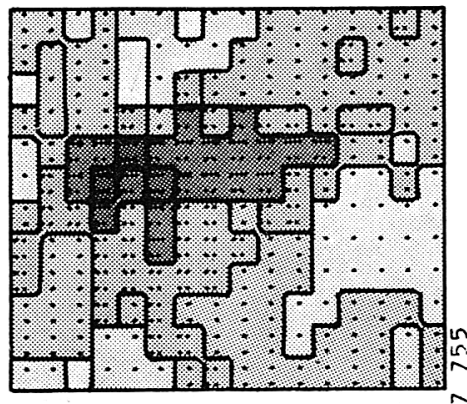

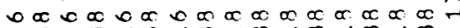
С゙
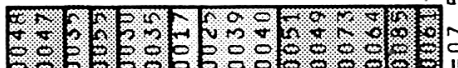

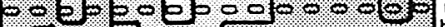

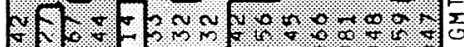

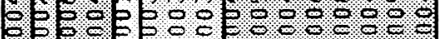
n

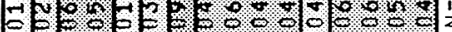

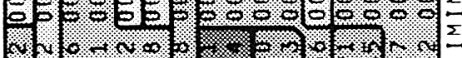

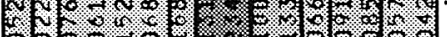

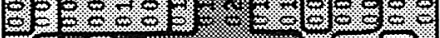

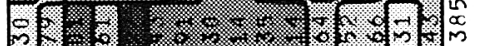
0 . 7 . of

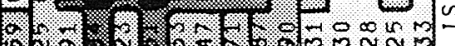
120120.000 \%

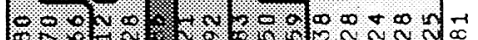
- O N

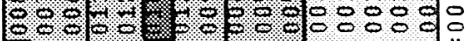

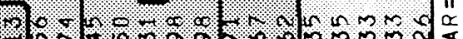

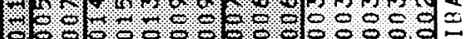

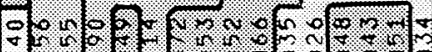

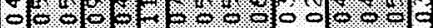
5) 5 .

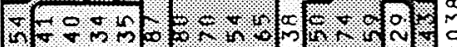

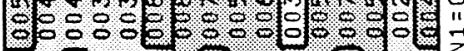

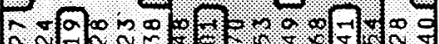

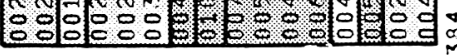

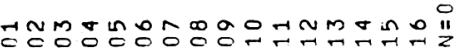




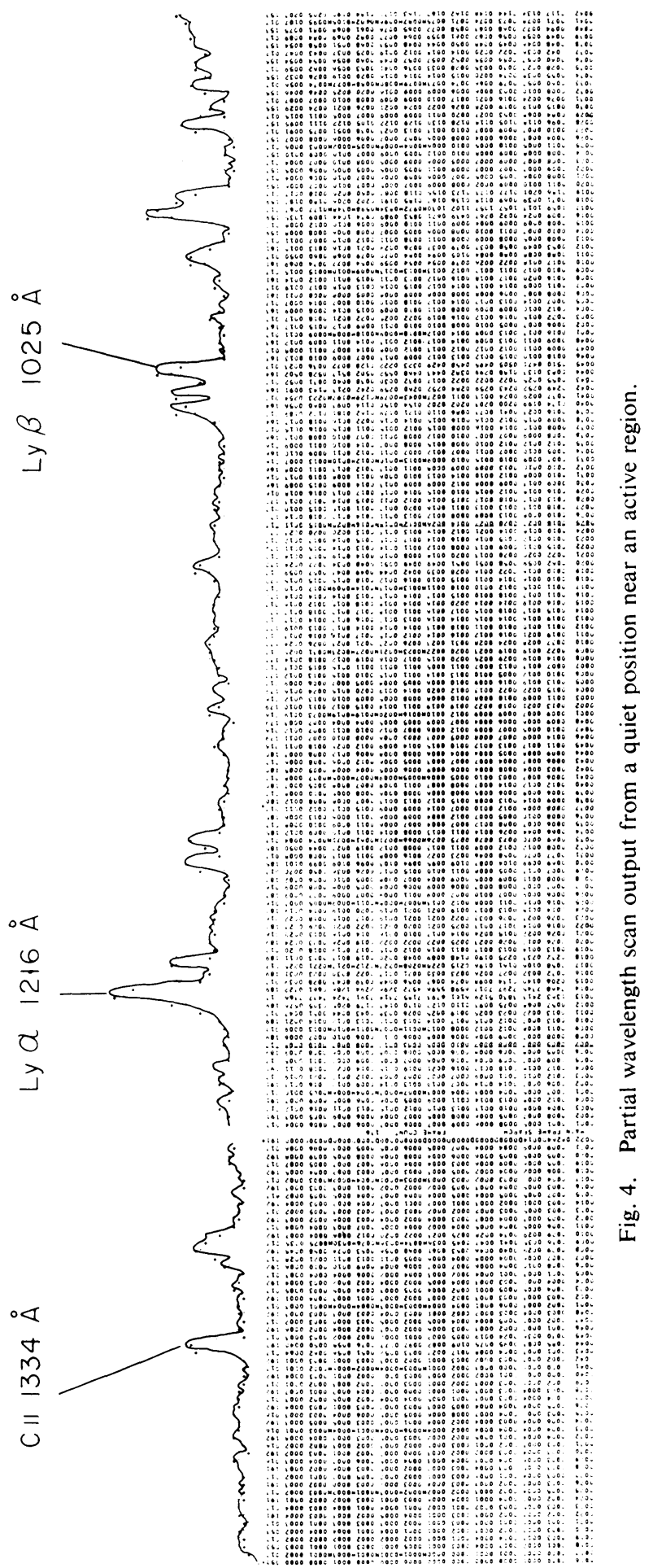


the prominent lines at the long wavelength end of the spectrum are shown in Figure 4. A data transmission dropout, which occurred infrequently, has been included to preserve the semblance of reality in our choice of 'typical' data for the illustrations.

In the procedure described above, namely the securing of wavelength scans from small regions on the solar disc such as multiple locations within active regions, holes in the corona, filaments, etc., it is necessary to allow for apparent movement of the features on the disc due to solar rotation. The time the data will be taken, the distance of the feature to be observed from the center of the disc, and the time at which the appropriate commands will be sent (or executed) must be taken into account in the planning process.

\section{Coordination with other Experiments}

In addition to the coordination described so far, a number of collaborative investigations were undertaken which required for their success simultaneous coordinated observations from the ground and from space. Among these were:

(i) A four-day search to observe the five-minute oscillation in the EUV. This program followed a quiet region on the Sun in lines of increasing ionization energy, while the same region was observed from the ground by Dr Wilcox at Mt. Wilson in September 1969.

(ii) A comparative study of the He 10830 line by Don Hall (Kitt Peak Observatory) over the active regions, while EUV observations were carried out in $\mathrm{HeI}$ lines and the Lyman continuum of hydrogen.

(iii) Observations of the total eclipse of March 7, 1970, for comparison with rocket observations in the EUV (Speer et al., 1970).

(iv) An investigation of the structure near the center of the solar disc to complement the observations of the $1400-1800 \AA$ region in a separate HCO rocket flight in September 1969.

(v) Comparative observations of the total intensity of the solar disc in lines of the EUV spectrum for calibration of the instrument against rocket flights by Hinteregger of AFCRL in August 1969, September 1969, and December 1969.

Collaborative investigations such as these, which simultaneously bring to bear the attributes of both space and ground-based facilities on a single objective enormously enhance the value of the separate space observations. No single instrument, in space or on the ground, embodies sufficient breadth of observational capacity to fully investigate the physical state on the Sun responsible for a given feature or event. The combination of capabilities, simultaneously applied to common features, is a rewarding and effective utilization of experiment capabilities. As solar ultraviolet research passes from the survey stage of the last decade into a period of search for the precise understanding of such problems as the processes of energy transfer in the solar atmosphere and the mechanisms giving rise to solar flares, among others, the necessity for the simultaneous application of many observational technique becomes indispensible. 


\section{Conclusion}

The attempt to control the complex observing program of a space experiment proved to be a significant undertaking and the whole process was remarkably successful. This was due in no small part to the NASA personnel at GSFC and remote ground stations who contributed to the operation ably and enthusiastically.

It is very easy to accumulate a great deal of data with a space experiment, but too frequently the experimenter comes close to drowning in continuing observations. However, as the options of spatial resolution and spectral resolution increase, the versatility of experiments also increases, and one must be very discriminating in the collection, and efficient in the cataloguing of data, so that the information applicable to a given observing objective can be extracted and processed further.

The capability to respond quickly to conditions on the Sun, the performance of the instrument, and most especially the information gained from a near real-time look at partially analysed data, completely justified the establishment of the data and communications link. In OSO I (EYE) (to be launched in 1972-1973) a similar link will be established with Boulder, Colorado, where the two experimenters of the pointed section will be situated. They will build on the experience gained here, and extend the system capability in the areas of data display and computer storage and access.

In EUV stellar astronomy, control of the star selection and experiment mode provides another example of real-time control of a scientific space experiment on a recent Orbiting Astronomical Observatory. The OAO orientation and experiment operation were controlled by personnel located in the OAO Control Center at GSFC and the data received from the spacecraft were displayed for partial analysis and operator guidance (Code et al., 1970).

An interesting advance in active and responsive control of solar experiments will be undertaken with the Skylab program, in which an astronaut will be present to operate the experiment. Pointing to preselected features on the Sun, and response to 'targets of opportunity' such as solar flares, should be significantly improved by this concept of manned operation. The astronaut will be provided with two on-board $\mathrm{H} x$ telescopes with video displays to point the various experiments, covering the visible, ultraviolet, and X-ray regions. The ability to operate the experiments while the astronauts are otherwise engaged or absent from the Skylab observatory has recently been incorporated into the program to provide in one mission the combined advantages of manned and unmanned capabilities. Video as well as digital data transmission will be part of the communication system.

\section{Acknowledgements}

The success of the instrument operation, the data acquisition, and control systems described here results from the combined efforts of persons in the engineering group of the Harvard Solar Satellite Project headed by Mr N. L. Hazen, and the many workers in the NASA Project Office, OSO Control Center, and Data Processing 
sections of GSFC. The planning, implementation, and operation of the Harvard data link included the participation of Drs A. K. Dupree, L. Goldberg, and W. H. Parkinson, as well as of many engineers, technicians, and students. The work has been sponsored by the National Aeronautics and Space Administration under contracts NASw-184 and NAS 5-9274.

\section{References}

Carson, D. G., Canfield, L. J., Ogne, M. L., Martin, D. C., and Reeves, E. M.: 1968, Astron.J. 73, S57. Code, A. D., Houck, T. E., McNall, J. F., Bless, R. C., and Lillie, C. F.: 1970, Astrophys. J. $161,377$. Macar, P. J., Rechavi, J., Huber, M. C. E., and Reeves, E. M.: 1970, Appl. Opt. 9, 581 ; (also available as Harvard College Observatory, Solar Satellite Project Report TR-9, October 1969).

Reeves, E. M. and Parkinson, W. H.: 1970, Astrophys. J. Suppl. 21, No. 181.

Reeves, E. M., Title, A., and Torgerson, D.: 1968, Astron. J. 73, S198.

Speer, R. J., Garton, W. R. S., Goldberg, L., Parkinson, W. H., Reeves, E. M., Morgan, J. F., Nicholls, R. W., Jones, T. J. L., Paxton, H. J. B., Shenton, D. B., and Wilson, R.: 1970, Nature 226, 249.

Title, A.: 1970, Harvard College Observatory, Solar Satellite Project Report TR-18. 